Series A

I. MATHEMATICA

397

\title{
RADII OF UNIFORM BOUNDEDNESS AND INDETERMINATION OF HOLOMORPHIC FUNCTIONS, AND EXAMPLES IN CONFORMAL MAPPING OF JORDAN REGIONS
}

BY

F. BAGEMIHL and J. E. McMILLAN

H E L I N K I 1967

S U O M A L A I NEN TIEDEAKA T EMIA

doi:10.5186/aasfm.1967.397 
Communicated 14 October 1966 by Olli Lehto and K. I. Virtanen 


\section{Introduction}

Let $C$ be the unit circle and $D$ be the open unit disk in the complex plane, and suppose that $E$ is a subset of $C$. In 1954, Bagemihl and Seidel [1] proved a general theorem on cluster sets which enabled them to derive the following result [1, p. 192, Theorem 3]:

A necessary and sufficient condition that there exist a function, holomorphic in $D$, that is uniformly bounded on the radii terminating in the points of $E$ but has no radial limit whatever, is that $E$ be nowhere dense on $C$.

They were then naturally led to ask the following question:

What is a necessary and sufficient condition that there exist a function, holomorphic in $D$, that is uniformly bounded on the radii terminating in the points of $E$ but has no radial limit at any point of $E$ ?

They made the conjecture (unpublished) at that time that such a condition was that $E$ be of Lebesgue measure zero on every arc of $C$ on which $E$ is everywhere dense.

That this is a reasonable conjecture is apparent from the following considerations. First of all, the necessity of the condition follows immediately from Fatou's theorem. The sufficiency of the condition is also readily established for two special cases: (i) $E$ is of measure zero, and (ii) $E$ is nowhere dense. In case (i), Lusin and Priwaloff have shown [9, pp. 156-159] that there exists a bounded holomorphic function in $D$ having no radial limit at any point of $E$. In case (ii), if $\bar{E}$ denotes the closure of $E$, then it follows from [1, p. 190, Corollary 2] that there exists a function, holomorphic in $D$, that is uniformly bounded on the radii terminating in the points of $\bar{E}$, but whose radial cluster set at every point of $\bar{E}$ is the unit circle. By an appropriate combination of these two examples, it is possible to prove the sufficiency of the condition for more general cases, but apparently not in general.

The purpose of the present paper is to establish the truth of the conjecture in its full generality. This is accomplished by constructing a Jordan curve $J \subset C \cup D$, with $E \subset J$, in such a way that the interior $\Delta$ of $J$ is star-shaped with respect to the origin $(0 \in \Delta)$, and under a conformal mapping of $\Delta$ onto the unit disk, the set $E$ corresponds to a set of measure 
zero on the unit circle. Then essentially the aforementioned example of Lusin and Priwaloff is transplanted to $\Delta$, and finally the desired holomorphic function is obtained in all of $D$ by means of an approximation that is also of independent interest.

The Jordan curve $J$ turns out to be useful for another purpose. For if we take $E$ in particular to be a perfect nowhere dense subset of $C$ of positive measure, and denote by $\Delta^{\prime}$ the exterior of $J$, then if $\Delta^{\prime}$ is mapped conformally onto the unit disk, it is not difficult to see that $E$ corresponds to a set of positive measure on the unit circle, whereas we have already noted that if $\Delta$ is mapped onto the unit disk, $E$ corresponds to a set of measure zero on the unit circle. Such an example has been given by Lohwater and Seidel [8], but in their example, no point of $E$ is finitely (or rectifiably) accessible from $\Delta$, whereas in our example every point of $J$ is rectilinearly accessible from $\Delta$ as well as from $\Delta^{\prime}$.

In the final section of this paper, an example is given of a Jordan curve $J$, with interior domain $\Delta$ and exterior domain $\Delta^{\prime}$, and a subset $H$ of $J$, with the property that $0 \in A, \Delta$ is star-shaped with respect to the origin, and in fact every point of $J$ is radially accessible (through $\Delta$ ) from the origin, and if $\Delta$ and $\Delta^{\prime}$ are mapped conformally onto the unit disk, the set $H$ corresponds to a set of measure 0 and $2 \pi$, respectively, on the unit circle.

We also give an example of a Jordan curve $J$, with interior domain 1 $(0 \in \Delta)$, and a subset $K$ of $J$ of positive area (two-dimensional Lebesgue measure), such that if $\Delta$ is mapped conformally onto the unit disk, the set $K$ corresponds to a set of measure zero on the unit circle. Such an example has been given by Lohwater and Piranian [6], but in their example, no point of $K$ is finitely accessible from $\Delta$, whereas in our example every point of $J$ is uniformly finitely accessible (through $\Delta$ ) from the origin.

\section{Outline of proof of conjecture}

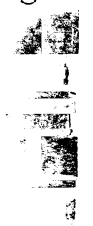

Theorem 1. Let $E$ be a subset of $C$. Then in order that there exist a function, holomorphic in $D$, that is uniformly bounded on the radii terminating in the points of $E$ but has no radial limit at any point of $E$, it is necessary and sufficient that $E$ be of Lebesgue measure zero on every arc on which it is everywhere dense.

Proof. The necessity of the condition clearly follows from Fatou's theorem.

Suppose now that $E$ satisfies the condition. Let $\left\{V_{n}\right\}$ be an enumeration of the (possibly only finitely many) components of $C-\bar{E}$. For each $V_{n}$, 
let $G_{n}$ be an open arc of $C$ such that $\bar{G}_{n} \subset V_{n}$, and set $G=\mathrm{U} G_{n}$. For an open arc $\gamma$ of $C$ and a real number $r(0<r<1)$, let

$$
S(\gamma, r)=\left\{\varrho e^{i_{\theta}}: r<\varrho<1, e^{i_{\theta}} \in \gamma\right\}, \quad \gamma(r)=\left\{r e^{i_{\theta}}: e^{i_{\theta}} \in \gamma\right\} .
$$

Choose a sequence $\left\{r_{m}\right\}$ such that $0<r_{m}<r_{m+1}<1(m \geqq 1)$ and $\lim r_{m}$ $=1$.

We shall construct a domain $A$, which will be $D$ minus certain of the sets $\bar{S}\left(G_{n}, r_{m}\right)$ and which will be bounded by a Jordan curve $J$, such that under a conformal mapping $\varphi$ of $\Delta$ onto $\left\{\left|w^{\prime}\right|<1\right\} \quad\left(=D^{\prime}\right)$, the subset $E$ of $J$ will correspond to a set $E^{\prime}$ on $\left\{\left|w^{\prime}\right|=1\right\} \quad\left(=C^{\prime}\right)$ of measure zero. Suppose for the moment that this has been done, and let $h$ be a function, bounded and bolomorphic in $D^{\prime}$, such that for each $\zeta \in E^{\prime}$ and for each arc $\sigma$ at $\zeta\left(\sigma \subset D^{\prime}\right.$ and $\sigma \cup\{\zeta\}$ is a Jordan arc), the diameter of the cluster set of $h$ on $\sigma$ at $\zeta$ is greater than 2 (the existence of such an $h$ can be established by means of a simple elakoration of the LohwaterPiranian version [7, p. 11, Theorem 4] of the Lusin-Priwaloff example [9, pp. 156-159], or most simply and directly by referring to an elegant version of this example given by W. Schneider [10]). Let $g(z)=h(\varphi(z))$ $(z \in \Delta)$. Then for each $\zeta \in E$, the oscillation of $g$ at $\zeta$ on the radius terminating at $\zeta$ is greater than 2 . We shall construct a function $f$, holomorphic in $D$, such that

$$
|f(z)-g(z)|<1 \text { if } z \in\left\{r e^{i_{\vartheta}}: 0 \leqq r<1, e^{i_{\theta}} \in E\right\} .
$$

It is clear that $f$ will then have the desired properties.

\section{Construction of $\Delta$}

We repeatedly use the following lemma of Löwner-Montel (see [3, p. 36]):

Suppose that $\Delta^{(j)}$ is the interior of a Jordan curve $J^{(j)}(j=1,2)$ and that $0 \in \Delta^{(1)} \subset \Delta^{(2)}$. Let $\varphi^{(j)}$ be a conformal mapping of $\Delta^{(j)}$ onto $D$ with $\varphi^{(j)}(0)=0, B$ be a Borel subset of $J^{(1)} \cap J^{(2)}$, and $B^{(j)}$ be the subset of $C$ that corresponds to $B$ under $\varphi^{(j)}$. Then $m\left(B^{(1)}\right) \leqq m\left(B^{(2)}\right)$ (where $m$ denotes Lebesgue measure).

Let $U$ be the interior (relative to $C$ ) of the closure $\bar{E}$ of $E$. Then $m(E \cap U)=0$. Thus $E \cap U$ corresponds to a set of measure zero under a conformal mapping onto $D^{\prime}$ of any domain which is $D$ minus certain of the sets $\bar{S}\left(G_{n}, r_{m}\right)$ and which is bounded by a Jordan curve. Hence we need to require of $\Delta$ only that $E-U$ correspond under $\varphi$ to a set of measure zero. 
The domain $\Delta$ will be defined as the intersection of a sequence $\left\{\Delta_{m}\right\}$. Let $m$ denote a fixed natural number. It is easy to see, by means of the extended maximum principle for harmonic functions, that we can let $\delta$ be a positive number such that, if $\gamma$ is an open arc of $C$ with length less than $2 \delta$, then the harmonic measure of $\gamma\left(r_{m}\right) \cup \gamma$ with respect to $S\left(\gamma, r_{m}\right)$ is less than $1 / m$ at each point of $\gamma\left(r_{m+1}\right)$. Let $U^{\prime}=C-\bar{G}$, and let $\left\{U_{n}^{\prime}\right\}$ be an enumeration of the (possibly only finitely many) components of $U^{\prime}$. Since each open arc of $C$ intersects $G \cup U^{\prime}$, we can determine a natural number $n_{0}$ such that each open arc of $C$ with length $\delta / 3$ intersects either $G$ or $\bigcup_{n=1}^{n_{0}} U_{n}^{\prime}$. Choose open $\operatorname{arcs} U_{n}^{\prime \prime}$ of $C\left(n=1, \ldots, n_{0}\right)$ such that $U_{n}^{\prime \prime} \supset \bar{U}_{n}^{\prime}$ and

$$
m\left(U^{*}\right)<1 / m, \text { where } U^{*}=\bigcup_{n=1}^{n_{0}}\left(U_{n}^{\prime \prime}-\bar{U}_{n}^{\prime}\right) .
$$

Choose a finite covering of $\bar{G}$ by open arcs (of $C$ ) intersecting $\bar{G}$, each with length less than $\delta / 3$, and let $n_{1}$ be a natural number such that each of these open ares intersects one of the sets $G_{1}, \ldots, G_{n_{1}}$. Let $n_{2}$ be a natural number such that $n_{2} \geqq n_{1}$ and for each $n=1, \ldots, n_{0}$, each component of $U_{n}^{\prime \prime}-\bar{U}_{n}^{\prime}$ intersects one of the sets $G_{1}, \ldots, G_{n_{2}}$. Set

$$
\Delta_{m}=D-\bigcup_{n=1}^{n_{2}} \bar{S}\left(G_{n}, r_{m}\right),
$$

and let $\varphi_{m}$ be a conformal mapping of $\Delta_{m}$ onto $D^{\prime}$ with $\varphi_{m}(0)=0$. We prove that under $\varphi_{m}, \bar{E}-U$ corresponds to a set on $C^{\prime}$ of measure less than $(2 \pi+1) / m$.

Let $G^{*}$ be the (possibly empty) union of the components of $C-\bigcup_{n=1}^{n_{2}} \bar{G}_{n}$ with length less than $2 \delta$. Denote by $u$ the harmonic measure of $G^{*}$ with respect to $\Delta_{m}$. If $\gamma$ is a component of $G^{*}$, then again by the maximum principle, at each $z \in S\left(\gamma, r_{m}\right), u(z)$ is less than the value at $z$ of the harmonic measure of $\gamma\left(r_{m}\right) \cup \gamma$ with respect to $S\left(\gamma, r_{m}\right)$; in particular, this holds for $z \in \gamma\left(r_{m+1}\right)$, so that $u(z)<1 / m$ if $z \in \gamma\left(r_{m+1}\right)$, because of the choice of $\delta$. Thus, by the maximum principle, $u(0)<1 / m$. Therefore, under $\varphi_{m}, G^{*}$ corresponds to a set on $C^{\prime}$ of measure less than $2 \pi / m$.

We now prove that all points of $\bar{E}-U$, with the exception of at most enumerably many, are in $U^{*} \cup G^{*}$. Note first that the set $(\bar{E}-U)-$ $(\bar{G}-G)$ is at most enumerable, since any accumulation point of $\bar{E}-U$ is in $\bar{G}-G$. Suppose that $\zeta$ is a point of $\bar{G}-G$ that is not an end point of any of the $\operatorname{arcs} G_{n}$ or $U_{n}^{\prime}$. We prove that $\zeta \in U^{*} \cup G^{*}$. Let $\gamma^{\prime}$ be an open arc of $C$ with length $\delta$ and one end point $\zeta$. Then, because of the choice of $n_{0}$, the open arc $\gamma^{\prime \prime}$ with length $\delta / 3$ that is the middle third of $\gamma^{\prime}$ 
intersects either $G$ or $\bigcup_{n=1}^{n_{0}} U_{n}^{\prime}$. If $\gamma^{\prime \prime}$ intersects $G$, then one of the sets $G_{1}, \ldots, G_{n_{1}}$ intersects $\gamma^{\prime}$. If $\gamma^{\prime \prime}$ intersects $U_{n^{\prime}}^{\prime}\left(1 \leqq n^{\prime} \leqq n_{0}\right)$, then, since $\zeta \notin \bar{U}_{n^{\prime}}^{\prime}$, either $\zeta \in U_{n^{\prime}}^{\prime \prime}-\bar{U}_{n^{\prime}}^{\prime}$, or one component of $\bar{U}_{n^{\prime}}^{\prime \prime}-\bar{U}_{n^{\prime}}^{\prime}$ is contained in $\gamma^{\prime}$ in which case one of the sets $G_{1}, \ldots, G_{n_{2}}$ intersects $\gamma^{\prime}$. Thus, either $\zeta \in U^{*}$, or each open arc with length $\delta$ and one end point $\zeta$ intersects one of the sets $G_{1}, \ldots, G_{n_{2}}$ (recall that $n_{2} \geqq n_{1}$ ), in which case $\zeta \in G^{*}$, since $\zeta \notin \bigcup_{n=1}^{n_{2}} \bar{G}_{n}$. Thus we have shown that all points of $\bar{E}-U$, with the exception of at most enumerably many, are in $U^{*} \cup G^{*}$.

Under $\varphi_{m}, U^{*} \cap(\bar{E}-U)$ corresponds to a set of measure less than $1 / m$, and $G^{*} \cap(\bar{E}-U)$ corresponds to a set of measure less than $2 \pi / m$. Hence $\bar{E}-U$ corresponds under $\varphi_{m}$ to a set of measure less than $(2 \pi+1) / m$. Let $\Delta=\bigcap_{m=1}^{\infty} \Delta_{m}$. Then the boundary of $\Delta$ is a Jordan curve $J, \quad \bar{E} \subset J$, and under a conformal mapping $\varphi$ of $\triangle$ onto $D^{\prime}$ with $\varphi(0)=0, \bar{E}-U$ corresponds to a set of measure zero. Since $m(E \cap U)=$ $0, E \cap U$ corresponds under $\varphi$ to a set of measure zero. Therefore $E$ corresponds under $\varphi$ to a set of measure zero.

\section{The approximation}

We suppose, for the sake of our notation and without loss of generality, that we can associate with each $G_{n}$ a natural number $m_{n}$ such that

$$
\Lambda=D-\cup \bar{S}\left(G_{n}, r_{m_{n}}\right) \text {. }
$$

For each $G_{n}$, choose an open arc $\gamma_{n}$ of $C$ such that $\bar{G}_{n} \subset \gamma_{n}$ and $\bar{\gamma}_{n} \subset V_{n}$, and let $\varrho_{n}$ be a positive number such that

$$
r_{m_{n}}-\frac{1}{n}<\underline{o}_{n}<r_{m_{n}} .
$$

Set $\Delta^{*}=D-\cup \bar{S}\left(\gamma_{n}, \varrho_{n}\right)$. Then the boundary of $\Delta^{*}$ is a Jordan curve $J^{*}$, which we orient counterclockwise. Let

$$
H=\left\{r e^{i_{\theta}}: 0 \leqq r<1, e^{i_{\ominus}} \in \bar{E}\right\} .
$$

Then $H \subset \Delta^{*}$. Choose a sequence $\left\{\tau_{n}\right\}$ such that $\varrho_{1}<\tau_{n}<\tau_{n+1}<1$ $(n \geqq 1), \quad \lim \tau_{n}=1$ and

$$
\tau_{n} \notin\left\{\varrho_{m}: m=1,2, \ldots\right\} \quad(n \geqq 1) .
$$


Set $\Gamma_{n}=\left\{|z|=\tau_{n}\right\} \cap \overline{\Lambda^{*}}$, and give the finitely many components of $\Gamma_{n}$ the counterclockwise orientation. Define

$$
C_{1}=J^{*} \cap\left\{|z| \leqq \tau_{1}\right\}, \quad C_{n}=J^{*} \cap\left\{\tau_{n-1} \leqq|z| \leqq \tau_{n}\right\} \quad(n>1) .
$$

Let the components of $C_{n}(n \geqq 1)$ have the orientation induced by $J *$. For any rectifiable oriented curve $\Gamma$ in $\Delta$, write

$$
I(\Gamma ; z)=\frac{1}{2 \pi i} \int_{\Gamma} \frac{g(\zeta)}{\zeta-z} d \zeta .
$$

If we approximate $I\left(C_{n} ; z\right)$ by means of a rational function having poles only on $C_{n}$, and apply Runge's pole-pushing lemma (see [2, p. 260]), we obtain a rational function $R_{n}(z)$, with poles only in the complement of $D$, such that

$$
\left|R_{n}(z)-I\left(C_{n} ; z\right)\right|<1 / 2^{n+1} \text { if }\left\{\begin{array}{l}
z \in H \text { and } n=1,2, \text { or } \\
z \in H \cup\left\{|z|<\tau_{n-2}\right\} \text { and } n>2 .
\end{array}\right.
$$

Let $f_{n}(z)=\sum_{j=1}^{n} R_{j}(z)+I\left(\Gamma_{n} ; z\right)\left(|z|<\tau_{n}\right)$. Then

$$
\left|f_{n}(z)-g(z)\right|<1 / 2 \text { if } z \in H \cap\left\{|z|<\tau_{n}\right\} .
$$

Note that if $n^{\prime}$ and $k$ are natural numbers with $n^{\prime}>n$, and if $|z|<\tau_{n}$, then

$$
\begin{aligned}
\left|f_{n^{\prime}+k}(z)-f_{n^{\prime}}(z)\right| & =\left|\sum_{j=n^{\prime}+1}^{n^{\prime}+t_{j}^{k}} R_{j}(z)+I\left(\Gamma_{n^{\prime}+k} ; z\right)-I\left(\Gamma_{n^{\prime}} ; z\right)\right| \\
& =\left|\sum_{j=n^{\prime}+1}^{n^{\prime}+k}\left(R_{j}(z)-I\left(C_{j} ; z\right)\right)\right|<1 / 2^{n} .
\end{aligned}
$$

Set $f(z)=\lim f_{n}(z) \quad(z \in D)$. Then $f$ is holomorphic in $D$ and $\mid f(z)-$ $g(z) \mid<1$ if $z \in H$. This completes the proof of Theorem 1 .

Remark. That this type of approximation is possible was learned by J. E. McMillan under the direction of G. R. MacLane. Stebbins [11] meanwhile obtained, independently and by a different method, a general approximation theorem which could be applied here to give the desired approximation.

\section{Examples in conformal mapping}

If we take $E$ to be a perfect nowhere dense subset of $C$ of positive measure, then it is clear from the foregoing construction of $\Delta$ and from $[8$, p. 138, Lemma 2] that the following is true. 
Theorem 2. There exists a Jordan curve $J$, with $J \subset C \cup D$, containing the origin in its interior $A$ which is star-shaped with respect to the origin, and having a closed set $E$ in common with $C$, such that if $\triangle$ and the exterior of $J$ are mapped conformally onto the unit disk, then $E$ corresponds to a set of measure zero and a set of positive measure, respectively, on the unit circle.

Remark. In the construction of $\Delta_{m}$ in Section 3, the points of $E-U$ that are near $U$ require special consideration, and it is evident how to simplify the argument considerably if $E$ is nowhere dense. It is easy to see, and it will be evident from the proof of Theorem 3, that we can also require in Theorem 2 that $J \cap C=E$ and that each point of $J$ be radially accessible from the origin.

A condensation process leads from Theorem 2 to the following result.

Theorem 3. There exist a Jordan curve $J$ containing the origin in its interior $\triangle$ such that every point of $J$ is radially accessible from the origin, and an $F_{\sigma}$ subset $H$ of $J$, with the property that if $\Delta$ and the exterior of $J$ are mapped conformally onto the unit disk, then $H$ corresponds to a set of measure zero and a set of measure $2 \pi$, respectively, on the unt circle.

Proof. Let $\left\{h_{n}\right\}$ be a sequence of positive real numbers such that $\Sigma h_{n}<1$. Associate with each $n$ a positive number $\delta_{n}$ such that if $r_{2}-r_{1} \geqq h_{n} / 2$ $\left(0<r_{1}<r_{2} \leqq 1\right)$ and $\Theta_{2}-\Theta_{1} \leqq \delta_{n} \quad\left(\Theta_{1}<\Theta_{2}\right)$, then the harmonic measure of

$$
\left\{r e^{i \ni}: r=r_{1}, r_{2} ; \Theta_{1}<\Theta<\Theta_{2}\right\}
$$

with respect to

$$
\left\{r e^{i \theta}: r_{1}<r<r_{2}, \Theta_{1}<\Theta<\Theta_{2}\right\}
$$

$i_{\mathrm{S}}$ less than $1 / n$ at each point of

$$
\left\{r e^{i \vartheta}: r=\left(r_{1}+r_{2}\right) / 2, \Theta_{1}<\Theta<\Theta_{2}\right\} .
$$

We define sequences $\left\{J_{n}\right\}$ and $\left\{F_{n-1}\right\}$ inductively as follows. Let $J_{1}=C$ and let $F_{0}=\varnothing$. Suppose that a Jordan curve $J_{n}$ has been defined such that

$$
J_{n} \subset\left\{1-\sum_{k=1}^{n-1} h_{k} \leqq|z| \leqq 1\right\},
$$

the interior $\Delta_{n}$ of $J_{n}$ is star-shaped with respect to 0 , and $J_{n}$ is a finite union of non-radial rectilinear segments and arcs of circles with center 0 ; and suppose that $F_{n-1}$ is a closed subset of $J_{n}$ which is nowhere dense on $J_{n}$. Denote by $\psi_{n}$ a conformal mapping of the exterior of $J_{n}$ onto $\left\{\left|z^{\prime}\right|<1\right\}$ 
such that $\psi_{n}(\infty)=0$, and let $F_{n}$ be a perfect set on $J_{n}$ such that $F_{n-1} \subset F_{n}, F_{n}$ is nowhere dense on $J_{n}$, and

$$
m\left(\psi_{n}\left(F_{n}\right)\right)>2 \pi-\frac{1}{n},
$$

where $\psi_{n}\left(F_{n}\right)$ denotes the subset of $\left\{\left|z^{\prime}\right|=1\right\}$ that corresponds to $F_{n}$ under $\psi_{n}$. Take $\left\{G_{k}\right\}$ to be an enumeration of the components of $J_{n}-F_{n}$, and set

$$
J_{n}^{*}=\left\{\left(r-h_{n}\right) e^{i_{\theta}}: r e^{i_{\vartheta}} \in J_{n}\right\}, G_{k}^{*}=\left\{\left(r-h_{n}\right) e^{i_{\vartheta}}: r e^{i_{\theta}} \in G_{k}\right\} \quad(k \geqq 1) .
$$

Let $k_{0}$ be a natural number and $U_{k}$ be an open subarc of $G_{k}^{*}$ $\left(k=1, \ldots, k_{0}\right)$ such that $\bar{U}_{k} \subset G_{k}^{*}$ and the following conditions (i), (ii), and (iii) are satisfied:

(i) The diameter of every component of each of the sets

$$
J_{n}^{*}-\bigcup_{k=1}^{k_{0}} \bar{U}_{k}
$$

and

$$
J_{n}-\bigcup_{k=1}^{k_{0}} \bar{G}_{k}
$$

is less than $h_{n} / 4$.

(ii) For each component $\gamma$ of the set (1), there exist $\Theta_{1}$ and $\Theta_{2}$ such that $0<\Theta_{2}-\Theta_{1} \leqq \delta_{n}$ and

$$
\gamma \subset\left\{r e^{i_{9}}: 0<r<1, \Theta_{1}<\Theta<\Theta_{2}\right\} \text {. }
$$

(iii) For each $k=1, \ldots, k_{0}$, the rectilinear segments $l_{k}$ and $r_{k}$ joining the left-hand (as viewed from the origin) end points of $G_{k}$ and $U_{k}$ and right-hand end points of $G_{k}$ and $U_{k}$, respectively, intersect $J_{n} \cup J_{n}^{*}$ only in their end points.

Denote by $D_{k}$ the interior of the Jordan curve that is the union of $G_{k}, \quad U_{k}, l_{k}$ and $r_{k}$. Set

$$
\Delta_{n+1}=\Delta_{n}-\bigcup_{k=1}^{k_{0}} \bar{D}_{k} .
$$

(Note that since $F_{n}$ is a perfect set, the distance between any two distinct domains $D_{k}$ is positive.) Let $J_{n+1}$ be the Jordan curve that is the boundary of $\Delta_{n+1}$. This completes the definition of $\left\{J_{n}\right\}$ and $\left\{F_{n-1}\right\}$.

We retain the notation used in the above definition, and take $\varphi_{n+1}$ to be a conformal mapping of $\Delta_{n+1}$ onto $\left\{\left|z^{\prime}\right|<1\right\}$ such that $\varphi_{n+1}(0)=0$. We now prove that

$$
m\left(\varphi_{n+1}\left(F_{n}\right)\right)<2 \pi / n
$$


Denote by $R$ an arbitrary component of the intersection of $\Delta_{n+1}$ and the exterior of $J_{n}^{*}$. The boundary of $R$ is the union of a component $\gamma_{1}$ of the set (1), a component $\gamma_{2}$ of the set (2), and two rectilinear segments. Write

$$
r_{1}=\sup \left\{|z|: z \in \gamma_{1}\right\}, \quad r_{2}=\inf \left\{|z|: z \in \gamma_{2}\right\} .
$$

Then (i) implies that $r_{2}-r_{1} \geqq h_{n} / 2$. Let $\Theta_{1}$ and $\Theta_{2}$ be such that $0<\Theta_{2}-\Theta_{1} \leqq \delta_{n}$ and

$$
\gamma_{1} \subset\left\{r e^{i_{\theta}}: 0<r<1, \Theta_{1}<\Theta<\Theta_{2}\right\} .
$$

Then $R \subset\left\{r e^{i_{\theta}}: 0<r<1, \Theta_{1}<\Theta<\Theta_{2}\right\}$. At each point of the circular arc

$$
R \cap\left\{r e^{i_{9}}: r=\left(r_{1}+r_{2}\right) / 2, \Theta_{1}<\Theta<\Theta_{2}\right\},
$$

the harmonic measure $u$ of the set (2) with respect to $\Delta_{n+1}$ is less than the harmonic measure of

$$
\left\{r e^{i \ni}: r=r_{1}, r_{2} ; \Theta_{1}<\Theta<\Theta_{2}\right\}
$$

with respect to

$$
\left\{r e^{i \ni}: r_{1}<r<r_{2}, \Theta_{1}<\Theta<\Theta_{2}\right\} .
$$

Thus $u(z)<1 / n$ if $z$ is on the arc (4), and it follows that $u(0)<1 / n$. Thus, since all points of $F_{n}$, with the possible exception of only finitely many, are in the set (2), we have established (3).

Define $\Delta=\cap \Delta_{n}$, and let $J$ be the Jordan curve that is the boundary of 4 . Then clearly every point of $J$ is radially accessible from 0 . If $H=\cup F_{n}$, then $H \subset J$. Let $\varphi$ and $\psi$ map the interior and exterior, respectively, of $J$ conformally onto $\left\{\left|z^{\prime}\right|<1\right\}$ so that $\varphi(0)=0$ and $\psi(\infty)=0$. By the lemma of Löwner-Montel (stated in Section 3),

$$
m\left(\varphi\left(F_{n}\right)\right) \leqq m\left(\varphi_{n+1}\left(F_{n}\right)\right) \quad(n \geqq 1) \text {. }
$$

Thus by (3),

$$
m(\varphi(H))=\lim m\left(\varphi\left(F_{n}\right)\right)=0 .
$$

On the other hand, by first subjecting the plane to the transformation $1 / z$ and then applying the lemma of Löwner-Montel, we see that

$$
m\left(\psi_{n}\left(F_{n}\right)\right) \leqq m\left(\psi\left(F_{n}\right)\right) \quad(n \geqq 1) .
$$

Hence

$$
2 \pi-\frac{1}{n}<m\left(\psi_{n}\left(F_{n}\right)\right) \leqq m\left(\psi\left(F_{n}\right)\right) \leqq m(\psi(H)) .
$$

Therefore, $m(\psi(H))=2 \pi$, and the proof of Theorem 3 is complete. 
A. I. 397

Remark. The foregoing constructions in Sections 3 and 5 could have been accomplished, less simply and directly, however, by combining geometrical arguments with a general theorem of Lavrentieff [5, p. 822, Theorem 1].

Theorem 4. There exist a Jordan curve $J$ containing the origin in its interior $\Delta$ such that every point of $J$ is uniformly rectifiably accessible through $\triangle$ from the origin, and a subset $K$ of $J$ of positive area, with the property that if $\Delta$ is mapped conformally onto the unit disk, then $K$ corresponds to a set of measure zero on the unit circle.

Proof. For an arbitrary (closed) isosceles right triangle $T$ and a positive number $\varepsilon$ that is less than the length of the hypotenuse $H$ of $T$, consider the open strip $S$ of width $\varepsilon$ such that the straight lines on the boundary of $S$ are perpendicular to $H$ and $T-S$ is the union of two congruent isosceles right triangles. Let $S_{\varepsilon}(T)$ be the intersection of $S$ with the union of $H$ and the interior of $T$. We shall choose a sequence $\left\{\varepsilon_{n}\right\}$ of positive numbers satisfying several restrictions, each of which will require that $\varepsilon_{n}$ be small. We first require $\left\{\varepsilon_{n}\right\}$ to be such that the following construction is possible.

Let $F_{1}$ be the (closed) isosceles right triangle that has a diameter of the unit circle as its hypotenuse and is contained in the closed upper half-plane. Suppose that a closed set $F_{n}$ has been defined so that its interior is the union of interiors of congruent (closed) isosceles right triangles $T_{n, j}$ $\left(j=1, \ldots, j_{n} ; j_{n}=2^{n-1}\right)$, and set

$$
F_{n+1}=F_{n}-\bigcup_{j=1}^{j_{n}} S_{\varepsilon_{n}}\left(T_{n, j}\right) .
$$

As in Knopp's triangle corsstruction (see [4, p. 204]), $\cap F_{n}$ is a Jordan arc $J^{\prime}$, and if the numbers $\varepsilon_{n}$ are sufficiently small, then $J^{\prime}$ has positive area. Let $J$ denote the Jordan curve

$$
J^{\prime} \cup\left\{e^{i \theta}: \pi \leqq \Theta \leqq 2 \pi\right\},
$$

and let $\Delta$ denote the interior of $J$. It is easy to see that each point of $J^{\prime}$ is accessible through $\Delta$ from the origin by a rectifiable curve that is composed of horizontal and vertical rectilinear segments and whose length is arbitrarily close to unity, and every other point of $J$ is radially accessible from the origin.

Define a sequence $\left\{A_{n}\right\}$ inductively as follows:

$$
\begin{aligned}
\Delta_{1} & =\left\{r e^{i_{9}}: 0<r<1, \pi<\Theta<2 \pi\right\} \cup S_{\varepsilon_{1}}\left(T_{1,1}\right), \\
\Delta_{n+1} & =\Delta_{n} \cup\left(\bigcup_{j=1}^{j_{2 n+1}} S_{\varepsilon_{2 n+1}}\left(T_{2 n+1, j}\right)\right) .
\end{aligned}
$$


Then $\Delta=\cup \Delta_{n}$. Let $J_{n}$ be the Jordan curve that is the boundary of $\Lambda_{n}$, and let $\varphi_{n}$ and $\varphi$ be conformal mappings of $\Delta_{n}$ and $\Delta$, respectively, onto $\left\{\left|z^{\prime}\right|<1\right\}$ with $\varphi_{n}(0)=0$ and $\varphi(0)=0$. Now fix the sequence $\left\{\varepsilon_{n}\right\}$ so that $J$ has positive area and for each $n$ and $k=1, \ldots, n$, the set

$$
J_{k} \cap\left(\bigcup_{j=1}^{j_{2 n}+1} S_{\varepsilon_{2 n+1}}\left(T_{2 n+1, j}\right)\right)
$$

corresponds under $\varphi_{k}$ to a set on $\left\{\left|z^{\prime}\right|=1\right\}$ of measure less than $1 / 2^{n}$. Note that each component of $J_{k} \cap \Delta$ is the intersection of $J_{k}$ and one of the sets $S_{\varepsilon_{2 n+1}}\left(T_{2 n+1, j}\right)(n \geqq k)$. Hence, under $\varphi_{k}, J_{k} \cap \Delta$ corresponds to a set of measure less than $1 / 2^{k-1}$, and $J_{k} \cap J$ corresponds to a set of measure greater than $2 \pi-\left(1 / 2^{k-1}\right)$. By the lemma of Löwner-Montel, $J_{k} \cap J$ corresponds under $\varphi$ to a set of measure greater than $2 \pi-\left(1 / 2^{k-1}\right)$. Therefore, under $\varphi$, the set

$$
E=\left(\bigcup_{k=1}^{\infty} J_{k}\right) \cap J,
$$

which has zero area, corresponds to a set of measure $2 \pi$, and the set $K=J-E$, which has positive area, corresponds to a set of measure zero. This completes the proof of Theorem 4.

University of Wisconsin-Milwaukee

\section{Bibliography}

[1] Bageminl, F. and Seidel, W., Some boundary properties of analytic functions. Math. Zeitschr. 61 (1954), 186-199.

[2] Behnke, H. and Sommer, F., Theorie der analytischen Funktionen einer komplexen Veränderlichen. 2d ed., Berlin, 1962.

[3] Gattegno, C. and Ostrowski, A., Représentation conforme à la frontière; domaines généraux. Paris, 1949.

[4] Hausdorff, F., Mengenlehre. 2d ed., Berlin, 1927.

[5] Lavrentieff, M., On some boundary problems in the theory of schlicht functions. Mat. Sbornik (N.S.) 1 (1936), $815-846$ (in Russian).

[6] Lohwater, A. J. and Piranian, G., Conformal mapping of a Jordan region whose boundary has positive two-dimensional measure. Michigan Math. J. 1 (1952), $1-4$.

[7] -》- The boundary behavior of functions analytic in a disk. Ann. Acad. Sci. Fenn. A I 239 (1957), 1-17.

[8] Lohwater, A. J. and SeIDel, W., An example in conformal mapping. Duke Math. J. 15 (1948), 137-143. 
[9] Lusin, N. and Priwaloff, J., Sur l'unicité et la multiplicité des fonctions analytiques. Ann. Sci. École Norm. Sup. (3) 42 (1925), 143-191.

[10] SchNeIDER, W., On the impossibility of sharpening the Fatou radial limit theorem (to appear).

[11] Stebbins, J. L., Spiral asymptotic values of functions meromorphic in the unit disk. Ph. D. Dissertation, Wayne State University, Detroit, 1965. 